\title{
Peginterferon Lambda-1a, a New Therapeutic for Hepatitis C Infection, from Bench to Clinic
}

\author{
Henrik Andersen, Jeff Meyer, Jeremy Freeman ${ }^{a}$, Sean E. Doyle, Kevin Klucher ${ }^{\mathrm{b}}$, \\ Dennis M. Miller ${ }^{c}$, Diana Hausman ${ }^{b}$ and Jan L. Hillson \\ ZymoGenetics/Bristol-Myers Squibb, Seattle, WA 98102, USA
}

\begin{abstract}
Chronic infection with hepatitis $\mathrm{C}$ virus (HCV) is estimated to affect approximately $3 \%$ of the world's population and cause 350,000 deaths each year. For a number of years, the standard of care has been combination therapy with recombinant alfa interferons-originally as native proteins but more recently as polyethyleneglycol-modified derivatives-and ribavirin, with the recent addition of an NS3 protease inhibitor for HCV genotype 1 . However, therapeutic alfa interferons are associated with a significant burden of treatment-limiting adverse events, including musculoskeletal and influenza-like symptoms, hematologic cytopenias, autoimmune disease, fatigue, and other neurologic events. In 2003, a team at ZymoGenetics (now a fully owned subsidiary of Bristol-Myers Squibb) and a second, independent group simultaneously identified a new class of interferons-the type III lambda interferons-with near-identical activity to the type I alfa interferons in hepatocytes but with an unrelated and less ubiquitous receptor. Subsequent evaluation of the type III interferon system demonstrated antiviral activity against HCV in vitro with limited activity in peripheral blood mononuclear cells and other nonhepatocyte cell types, supporting its development as a potentially better-tolerated therapy for viral hepatitis. Peginterferon lambda-1a (Lambda) is an investigational type III therapeutic agent originally developed at ZymoGenetics that is currently in Phase 3 studies for the treatment of HCV. In this review, we describe the selection of the Lambda molecule and its preclinical and early clinical development, and how the resulting data have helped to establish the differentiated safety profile for Lambda-with fewer influenza-like and musculoskeletal symptoms and less hematologic toxicity than the alfa interferons-that was seen in later studies.

(c) 2013 The Second Affiliated Hospital of Chongqing Medical University. Published by XIA \& HE Publishing Ltd. All rights reserved.
\end{abstract}

\section{Keywords: Discovery; HCV; Interferon $\lambda$.}

Abbreviations: $\beta 2 \mathrm{M}, \beta 2$-microglobulin; $\mathrm{GT}$, genotype; HBV, hepatitis $B$ virus; $\mathrm{HCV}$, hepatitis C virus; HLA, human leukocyte antigen; IFN, interferon; IL, interleukin; IL28RA, interleukin-28 receptor $\alpha$; ISG, interferon-stimulated gene; NOEL, no observed effect level; PBMC, peripheral blood mononuclear cell; PEGIFN, peginterferon; PK, pharmacokinetics; Rh, hydrodynamic radius; STAT, signal transducer and activator of transcription.

Received: 30 August 2013; Revised: 11 November 2013; Accepted: 15 November 2013

DOI of original article: 10.14218/JCTH.2013.00014.

Correspondence to: Jan L. Hillson, ZymoGenetics, 1201 Eastlake Avenue East, Seattle, WA 98102, USA. Tel: +1-206-617-4822, E-mail: jan.hillson@bms.com

${ }^{a}$ Currently at EMD Serono, Billerica, MA, USA

${ }^{\mathrm{b}}$ Currently at Oncothyreon Inc., Seattle, WA, USA

${ }^{c}$ Currently at CPTXBio, Woodinville, WA, USA

\section{Introduction}

Chronic hepatitis $\mathrm{C}$ virus (HCV) infection affects approximately 170 million people worldwide, ${ }^{1}$ with an estimated 350,000 deaths each year from long-term complications such as cirrhosis and hepatocellular carcinoma. ${ }^{2}$ Recombinant type I interferons (IFNs), specifically IFN alfa-2a and IFN alfa- $2 b$, have been used alone or in combination with the nucleoside analog ribavirin (and, more recently, with or without an NS3 protease inhibitor) to treat chronic viral hepatitis for more than 20 years. Combination treatment is potentially curative in a significant proportion of patients infected with HCV. However, therapeutic IFN alfa carries a significant burden of system-wide adverse events, which reflects the ubiquitous distribution of its receptor. ${ }^{3}$ Hematologic abnormalities from IFN alfa-associated myelosuppression are a particular problem in HCV treatment, and neutropenia, thrombocytopenia, and especially IFN-exacerbated, ribavirin-associated hemolytic anemia are major causes of dose adjustment and discontinuation. ${ }^{3}$ The use of NS3 protease inhibitors against HCV genotype (GT) $1^{4}$ further adds to an already high adverse event rate. ${ }^{5-7}$

In 2003, two groups-Sheppard et al. ${ }^{8}$ from the ZymoGenetics discovery team and Kotenko et al. ${ }^{9}$-independently identified a new family of IFNs with their own receptor complex, designated the type III $\lambda$ IFNs. As this new cytokine system became better understood a firm rationale for its therapeutic development in viral hepatitis emerged, and 10 years later peginterferon (PEG-IFN) lambda-1a (Lambda) is now in Phase 3 studies in HCV. Here, we briefly review the preclinical and early clinical development of this novel biologic.

Discovery and early preclinical evaluation of the $\lambda$ IFNs: tissue specificity and in vitro activity

A number of excellent reviews of the $\lambda$ IFNs and their receptor are available. ${ }^{10-12}$ The type III family was initially identified by analysis of human genomic sequence data to identify potential new cytokines, and comprises three multi-exon genes on chromosome 19 (19q13.13 contig AC011445.6). Two interleukin (IL)-28 genes, IL28A and IL28B, comprise six exons each and encode two near-identical ( $96 \%$ amino acid homology) secreted proteins, designated IFN $-\lambda 2$ and IFN $-\lambda 3$, respectively. IL 29 is a five-exon gene encoding IFN- $\lambda 1$, which is $81 \%$ homologous to IFN- $\lambda 2$. In contrast to the similarities within the type III group, there is less than $20 \%$ homology between any IFN $\lambda$ and IFN $\alpha$. The transmembrane receptor for the IFN $\lambda$ proteins is a heterodimer that is structurally 
distinct from the type I receptor and consists of a unique $\alpha$ subunit, IL-28 receptor $\alpha$ (IL28RA), combined with a ubiquitous $\beta$ subunit (IL-10 receptor $\beta$; IL10RB) that also forms part of the receptor complexes for IL-10, IL-22, and IL-26.

The distribution of the type III receptor complex differs significantly from that of the type I complex. At the mRNA level, type III receptors have been detected in most tissues other than the brain and central nervous system. ${ }^{13,14}$ Within tissues, however, type III receptors appear to be expressed in a subset of type I-responsive cells. Induction of IFNstimulated genes (ISGs) by type III IFNs is observed primarily in epithelial cells and appears to be highest in organs with a high epithelial component. ${ }^{15}$ Within cells expressing both receptors, IFN $\lambda$ and IFN $\alpha$ appear to present almost identical patterns of signal transducer and activator of transcription (STAT) phosphorylation via a shared Janus kinase-STAT pathway and similarly near-identical patterns of gene regulation (via the ISG factor 3 activation complex) by microarray analysis. ${ }^{13,16}$

IL28RA is expressed on primary hepatocytes, which are epithelial-like cells, and on hepatocyte-derived cell lines but not on other cells present in the liver. ${ }^{13}$ In peripheral blood, functional IL28RA is produced in plasmacytoid dendritic cells, in which exogenous type III IFNs have been shown to elevate Herpes simplex virus-induced production of both type I and type III molecules. ${ }^{17}$ IL28RA mRNA is expressed in B lymphocytes, but these cells show little or no induction of STAT phosphorylation when treated with type III IFNs. ${ }^{13,14,18}$ In stark contrast to the type I complex, other peripheral immune cells show little or no expression of type III receptors or response to type III molecules. ${ }^{13,14}$

Type III antiviral activity was initially demonstrated in vitro in protection assays using vesicular stomatitis virus in the epithelial cell lines A549 (alveolar adenocarcinoma), HaCaT (immortalized keratinocyte), and HT29 (colorectal adenocarcinoma), and using encephalomyocarditis virus in HT29 and HepG2 (hepatocellular carcinoma) cells. ${ }^{8,9}$ Activity in vitro against hepatitis $B$ virus (HBV) and HCV was established by ZymoGenetics using a stable HBV-infected HepG2 line and a stable subgenomic HCV replicon-transfected $\mathrm{HuH7}$ cell line, respectively, ${ }^{13}$ and by others using a variety of viral infection and genomic/subgenomic replicon assays. ${ }^{16,19,20}$

Selection and preclinical development of Lambda as a therapeutic candidate for HCV infection

A new IFN class with similar hepatic activity to IFN $\alpha$ but less unwanted activity elsewhere was quickly recognized to have potential utility as a treatment for viral hepatitis. Despite initial concerns about how well cell culture infection models would predict human efficacy, and the theoretical possibility of peripheral viral reservoirs unsusceptible to type III IFN activity, the potential safety and tolerability advantages inherent in the reduced extrahepatic activity of the type III class argued for its clinical development.

Given that all three of the type III cytokines had demonstrated acceptable activity in multiple bioassays, the decision to develop IFN- $\lambda 1$ as the clinical candidate, rather than either of the IL28 gene products, was primarily driven by manufacturing advantages. The three type III polypeptides are relatively simple and active in monomeric form, and although human IFN- $\lambda 1$ is glycosylated (unlike the other two members of the family), the glycan found on the mature secreted protein does not appear to be necessary for biologic activity. A standard Escherichia coli expression system was therefore developed, similar to those used to produce other cytokine therapeutics. However, all three members of the type III family contain an odd number of cysteine residues in the mature polypeptide, and E. coli expression followed by refolding resulted in a heterogeneous (and difficult to separate) mixture of protein isoforms with differing configurations of intramolecular disulfide bridges. Both IFN- $\lambda 2$ and IFN- $\lambda 3$ have seven cysteines at positions $16,48,50,115$, 148,167 , and 174 in the mature native protein, which give rise to nine combinations of bridged pairs in the expressed protein. By contrast, IFN- $\lambda 1$ has only five cysteines (at positions $15,49,112,145$, and 171) and three combinations of bridged pairs: C15-C112, C49-C145, and C112-C171. A conservative substitution of serine for the cysteine at position 171 , together with a short N-terminal deletion and addition of an $\mathrm{N}$-terminal methionine, resulted in an IFN- $\lambda 1$ expression construct that generated a single, readily purified synthetic protein with two fixed disulfide bridges and full biologic activity.

Based on previous experience with therapeutic PEG-IFN alfa indicating that more prolonged exposure improves response, ${ }^{21,22}$ it was considered important that recombinant IFN- $\lambda 1$ be modified to improve its pharmacokinetics (PK). The recombinant protein has a similar molecular weight $(19.6 \mathrm{kDa})$ to the unpegylated IFN alfa drugs and it was assumed that, as for the PEG-IFN alfa constructs, similar pegylation of IFN- $\lambda 1$ would decrease renal clearance and increase half-life. "Similar pegylation" in this context refers to comparisons of the hydrodynamic radius ( $\mathrm{Rh}$ ) and apparent molecular weight of the constructs rather than to the molecular weight of the PEG modifier, because the diverse range of PEG structures and attachment chemistries available make molecular weight alone a poor predictor of a pegylated molecule's properties.

Both of the current PEG-IFN alfa therapeutics for viral hepatitis are mixtures of monopegylated positional isomers in which the polymers are covalently linked to one of several internal residues. ${ }^{23}$ PEG-IFN alfa-2b (PegIntron; Merck) has a 12-kDa linear PEG moiety attached to a number of different internal amino acids via a succinimidyl carbonate process, although with the majority $(>50 \%)$ attached at a single histidine at position 34. ${ }^{24}$ PEG-FIN alfa-2a (Pegasys; Roche/ Genentech) uses a highly lysine-specific $N$-hydroxysuccinimide process for its $40-\mathrm{kDa}$ branched PEG moiety, which results in four major positional isomers and a further seven minor ones. ${ }^{25}$ The weight-based dosing of PEG-IFN alfa-2b is held to be the result of the relatively small PEG attachment. However, the heterogeneity of the modified products and the branched structure of the 40-kDa attachment for the PEG-IFN alfa-2a drug-which might be expected to confer a quite different apparent mass than a linear polymer of the same weight-makes it difficult to draw a priori assumptions about optimal pegylation for a similarly sized protein.

More recent pegylation chemistries reduce heterogeneity by more precisely targeting the $\mathrm{N}$-terminal amino group for modification. Ultimately, an mPEG-propionaldehyde reductive alkylation process ${ }^{26}$ was used to attach a $20-k D a$ linear PEG to the N-terminal methionine of the recombinant protein. The N-terminal attachment mitigated concerns associated with the $\mathrm{N}$-terminal methionylation as well as the short $\mathrm{N}$ terminal deletion of the native human sequence. Evaluation of Rh as a surrogate for apparent molecular weight using sizeexclusion chromatography equipped with multi-angle light 
scattering and quasi-elastic light scattering demonstrated that recombinant synthetic IFN- $\lambda 1$ with a $20 \mathrm{kDa} N$-terminal linear PEG attachment (hereafter referred to as "Lambda") had an Rh of $4.9 \mathrm{~nm}$, compared with $4.7 \mathrm{~nm}$ for PEG-IFN alfa$2 \mathrm{~b}$ and $5.2 \mathrm{~nm}$ for PEG-IFN alfa-2a. The assumption that similar Rh and thereby similar apparent mass would result in broadly similar PK between Lambda and PEG-IFN alfa has been confirmed in both animal and human studies. ${ }^{18,27-29}$

Preclinical and nonclinical development of Lambda: safety, PK, and pharmacodynamics

Although both Lambda and the type I IFNs were established to be biologically and antivirally active in hepatocytes, on a mole-for-mole basis type III IFNs generally promoted lower levels of STAT phosphorylation and gene induction in vitro ${ }^{13,16}$ than type I IFNs, and exhibited lower anti-HBV or anti-HCV activity in cell culture models in several studies. ${ }^{16,19}$ Although there are differences in the kinetics of both signaling and gene modulation between the type I and type III systems that complicate the interpretation of single time-point comparisons, ${ }^{16,30}$ these observations raised concerns regarding the relative in vivo potency of Lambda compared with PEGIFN alfa that would not be resolved until early clinical data became available.
Despite these early questions about its potential efficacy, early in vitro assessment of Lambda's hematologic activity was very promising from the perspective of its likely in vivo safety profile. No effect of Lambda on the proliferation of human bone marrow mononuclear cells was seen over a 2-log concentration range (Fig. 1). ${ }^{31}$ In contrast, and as expected, PEG-IFN alfa controls demonstrated concentration-dependent inhibition of erythroid and myeloid colony formation consistent with the known myelosuppressive effects of the alfa IFNs in vivo. Furthermore, when human peripheral blood mononuclear cells (PBMCs) were treated with Lambda or PEG-IFN alfa-2a, only the type I IFN stimulated IL-6 release (Fig. 2). These observations, consistent with the known absence (or low levels) of functional type III receptors in bone marrow progenitors and peripheral blood, strongly suggested that Lambda would not be associated with the common hematologic toxicities of PEG-IFN alfa. Furthermore, the lack of PBMC cytokine stimulation suggested that Lambda treatment was unlikely to cause influenza-like symptoms or other sequelae of systemic immune activation seen with the type I drugs.

The major nonclinical in vivo model for Lambda was the cynomolgus monkey (Macaca fascicularis), which had already been well established in studies of recombinant human IFN $\alpha .^{32,33}$ The initial feasibility of the cynomolgus model for evaluating human type-III cytokines was established by

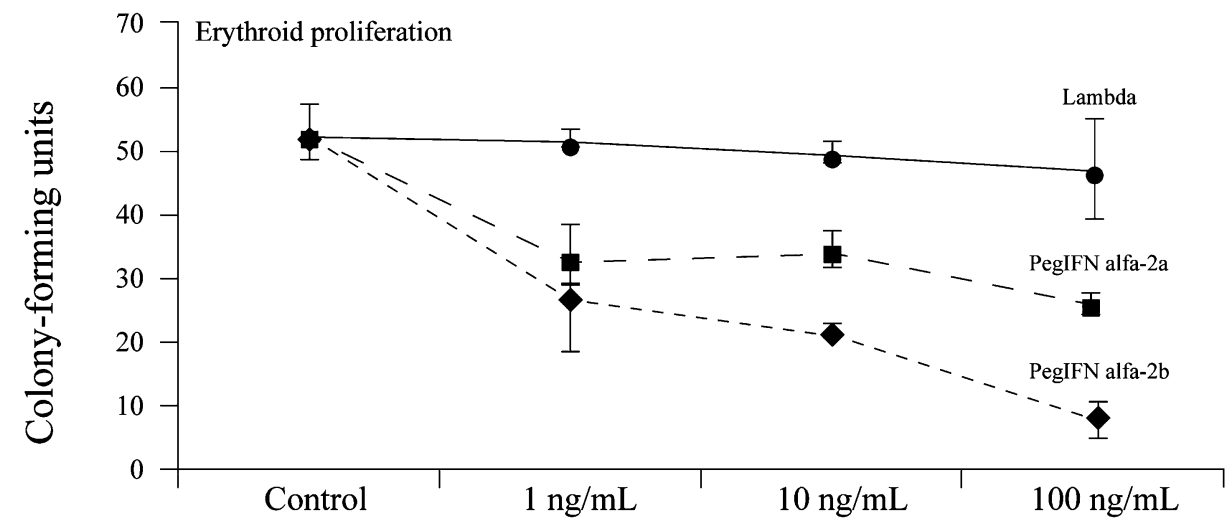

Concentration

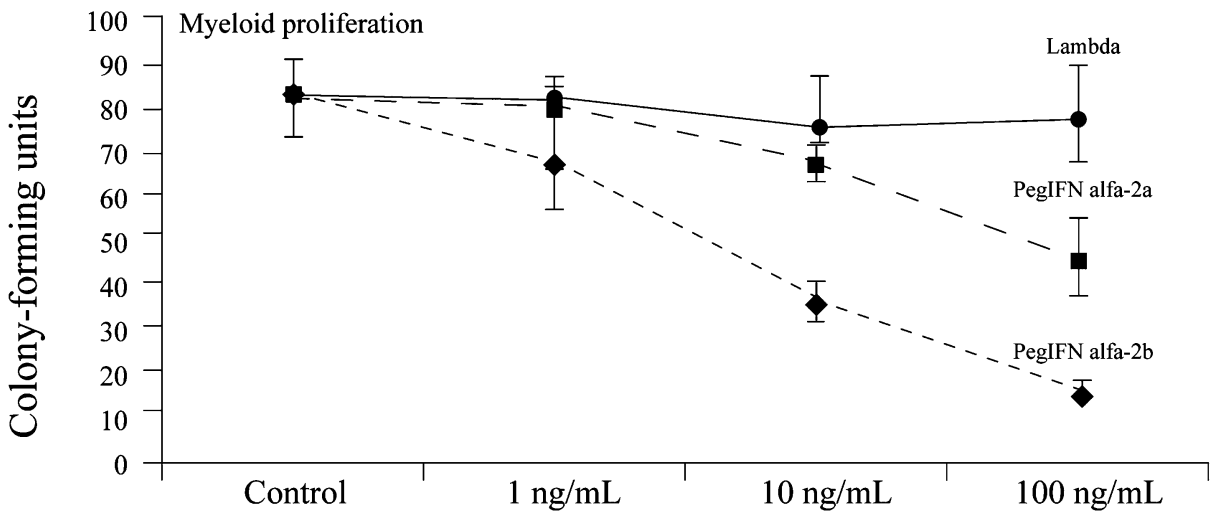

Concentration

Fig. 1. Early preclinical data showing the effect of peginterferon alfa and peginterferon lambda-1a on the proliferation of human bone marrow mononuclear cells 
Anderson H. et al: Peginterferon lambda for hepatitis C

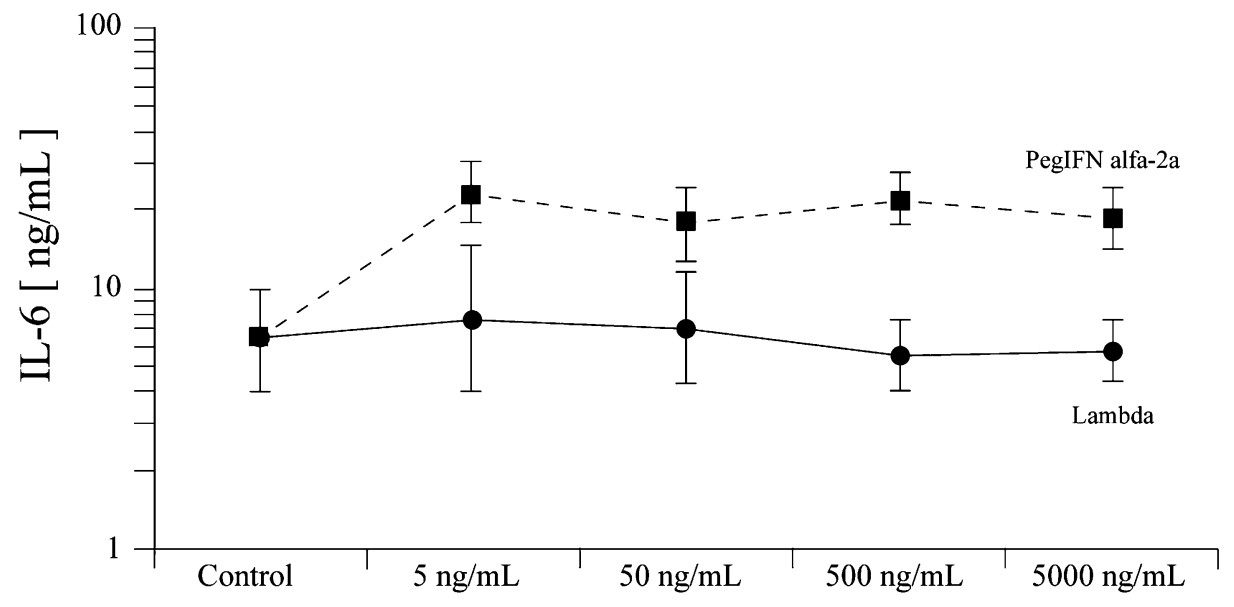

Concentration

Fig. 2. Early preclinical data showing the effect of peginterferon alfa-2a and peginterferon lambda-1a on interleukin-6 release in human peripheral blood mononuclear cells

\section{1}

Human: MAGPERWGPL LLCLLQAAPG RPRLAPPQNV TLLSQNFSVY LTWLPGLGNP QDVTYFVAYQ Cynomolgus: MAGPERWGPL LLCLLQAAPG RPRLAPPQNV TLLSRNFSVY LTWLPGLGNP QDVTYFVAYQ

61

120

Human: SSPTRRRWRE VEECAGTKEL LCSMMCLKKQ DLYNKFKGRV RTVSPSSKSP WVESEYLDYL Cynomolgus: SSPTPRRWRK VEECAGTKEL LCSMMCLKKQ DLYNKFKGRV QTVSPSSKSP WVESEYVDYL

121

180

Human: $\quad$ FEVEPAPPVL VLTQTEEILS ANATYQLPPC MPPLDLKYEV AFWKEGAGNK TLFPVTPHGQ Cynomolgus: FEVEPAPPVL VLTQTEEILS ANATYQLPPC MPPLDLKYEV AFWKEGAGNK TLFPVTPHGQ

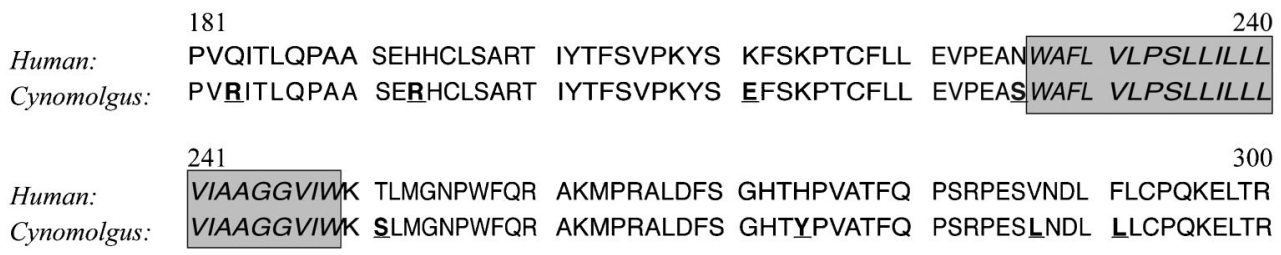

$301 \quad 360$

Human: GVRPTPRVRA PATQQTRWKK DLAEDEEEE. .DEEDTEDGV SFQPYIEPPS FLGQEHQAPG Cynomolgus: GVRPTPRVRA PATQQAGWKK DLAEDEDEEE EEEEDTEDGV SFQPYIEPPS FLGQEHQVPG

$361 \quad 420$

Human: HSEAGGVDSG RPRAPLVPSE GSSAWDSSDR SWASTVDSSW DRAGSSGYLA EKGPGQGPGG Cynomolgus: HSEVGGVDSG RPRAPLLPGE GSSAWDSSDR SWASTVDSSW DRAGSSGYLA EKGPGQGPGG $421 \quad 480$ Human: DGHQESLPPP EFSKDSGFLE ELPEDNLSSW ATWGTLPPEP NLVPGGPPVS LQTLTFCWES Cynomolgus: DGHQEPLPPP EFSTDSGFLE ELPKDDLLSSW ATWGTLPPE $\underline{\mathbf{S}}$ NLVPGGPPVS LRTLTFCWES $481 \quad 525$

Human: $\quad$ SPEEEEE... ARESEIEDSD AGSWGAESTQ RTEDRGRTLG HYMAR Cynomolgus: SPEEEEEEEE ARESEIEDSD AGSWGAESTQ RTEERGRTRG HYMAR

Fig. 3. Amino acid sequence alignment of human and cynomolgus monkey interleukin-28 receptor $\boldsymbol{\alpha}$. Cynomolgus differences from human residues are underlined. The boxed region shows the transmembrane domain. 
immunohistochemical confirmation of liver receptor expression with anti-human IL28RA, followed by confirmation of hepatocyte ISG induction by Lambda at both the mRNA and protein levels. ${ }^{18}$ Cloning and sequencing of cynomolgus IL28RA at ZymoGenetics identified a 525-residue protein with close amino acid homology (approximately 94\% similarity; Fig. 3) to the 520-residue human receptor subunit, accounting for both its strong cross-reactivity with the antihuman antibody and the activity of the human Lambda construct. Subsequent single- and multiple-dose studies in the cynomolgus model included noncompartmental PK and exposure simulations, blood versus liver activity of Lambda and serum biomarker analyses, and Good Laboratory Practice toxicology studies.

A single intravenous dose of Lambda over the range of $0.03-3.0 \mathrm{mg} / \mathrm{kg}$ in cynomolgus monkeys resulted in approximately dose-proportional exposures and a terminal half-life of 23 hours, which were consistent with the findings for other, similarly sized PEG-IFNs. ${ }^{18}$ Cynomolgus liver biopsies obtained after several days of dosing with Lambda or IFN alfa-2b showed strong induction of the myxovirus resistance $(M X A)$ and protein kinase R (PKR) ISG mRNAs and expression of $2^{\prime}, 5^{\prime}$-oligoadenylate synthetase protein by both IFNs. ${ }^{18}$ The lack of a pharmacodynamic effect for Lambda in immune cells observed ex vivo was confirmed in vivo in the cynomolgus model. No upregulation of MXA or PKR was seen in unfractionated PBMCs extracted 4 hours after single-dose Lambda administration, and no induction of class I human leukocyte antigen (HLA) expression was seen in fractionated PBMC subsets. By contrast, a control dose of unpegylated IFN alfa- $2 \mathrm{~b}$ resulted in significant PBMC upregulation of both $M X A$ and $P K R$ transcripts in unfractionated PBMCs and elevated HLA expression in all PBMC subsets. Only $\beta 2$-microglobulin $(\beta 2 \mathrm{M})$ - a component of class I HLA molecules-was identified as a serum marker for Lambda activity, with both IFN alfa-2b and all Lambda doses above $0.015 \mathrm{mg} / \mathrm{kg}$ resulting in significant increases in serum $\beta 2 \mathrm{M}$ concentrations. Induction of $\beta 2 \mathrm{M}$ was higher with IFN alfa-2b than with Lambda, consistent with the larger number of type I-responsive cells. Notably, Lambda, unlike IFN alfa-2b, had no effect on levels of serum neopterin, an established marker of exogenous type I IFN activity in leukocytes. The use of $\beta 2 \mathrm{M}$ as a peripheral pharmacodynamic marker of Lambda activity allowed initial estimates for human dosing to be made on the basis of the $0.015 \mathrm{mg} / \mathrm{kg}$ no observed effect level (NOEL) determined in the monkey model.

Good Laboratory Practice safety studies in cynomolgus monkeys (described briefly in Miller et al. ${ }^{34}$ ) corroborated the in vivo implications of the PBMC and bone marrow data for Lambda described above. Repeated Lambda dosing at $2.5 \mathrm{mg} / \mathrm{kg}$ twice weekly resulted in no mortality, "sickness behavior," change in body weight, loss of red or white blood cells, decrease in serum protein, bone marrow hypocellularity, or thymus atrophy.

\section{Phase 1/2a clinical development for HCV}

Given the consistency of the safety data but the uncertainty at the time around Lambda's antiviral efficacy, there was some internal discussion about whether the first-in-human study for Lambda should take a proof-of-concept approach in $\mathrm{HCV}$-infected patients. Although initial studies of this type are not uncommon in some branches of medicine, and have the advantage of addressing the basic viability of a candidate in its target population, it was ultimately decided to adopt the more standard approach of a single-dose, first-in-human safety assessment in healthy volunteers.

The subsequent multiple-dose proof-of-concept study evaluated Lambda's virologic activity from weekly doses over 4 weeks of administration in patients with HCV infection. The population selected comprised patients who had previously relapsed after demonstrating a decline in circulating viral load following at least 12 weeks of treatment with pegylated or unpegylated IFN alfa with ribavirin. At the time of enrolment, patients in this category were considered to have demonstrated potential responsiveness to IFN, as opposed to nonresponders; unlike treatment-naive patients, however, they did not have options for curative therapy. A single treatment-naive cohort of seven patients was also enrolled.

Subsequently, a follow-on Phase 2a study directly compared Lambda and PEG-IFN alfa, both with ribavirin, over longer-term administration in treatment-naive patients.

\section{Phase 1a: healthy volunteers}

The first-in-human safety study of Lambda ${ }^{29,31}$ was a singleascending-dose study comprising four cohorts of six volunteers each. Participants were randomized $5: 1$ to receive a single subcutaneous dose of Lambda or placebo, with dosing based on scaling of the $15 \mu \mathrm{g} / \mathrm{kg}$ NOEL in cynomolgus monkeys for induction of serum $\beta 2 \mathrm{M}(5 \mu \mathrm{g} / \mathrm{kg}$ equivalent human dose). Overall, 20 volunteers were enrolled and received $0.5 \mu \mathrm{g} / \mathrm{kg}(\mathrm{n}=5$ receiving Lambda), $1.5 \mu \mathrm{g} / \mathrm{kg}$ ( $\mathrm{n}$ $=5), 5.0 \mu \mathrm{g} / \mathrm{kg}(\mathrm{n}=5)$, or $7.5 \mu \mathrm{g} / \mathrm{kg}(\mathrm{n}=2 ;$ maximum tolerated dose)

Lambda was well tolerated overall, with no significant hematologic changes (Fig. 4) other than mild dose-dependent transient decreases in fibrinogen levels (also observed in the monkey model) and similar transient mild asymptomatic increases in prothrombin time. Two participants receiving $5.0 \mu \mathrm{g} / \mathrm{kg}$ had transient grade 2 elevations in alanine aminotransferase levels, and one of two participants enrolled at $7.5 \mu \mathrm{g} / \mathrm{kg}$ had a transient dose-limiting grade 3 alanine aminotransferase elevation. Lambda PK were dose dependent, with a time to maximal concentration that varied between 8 and 24 hours and an estimated terminal half-life of 50-70 hours. Significant elevations in serum $32 \mathrm{M}$ levels were observed at doses of $1.5 \mu \mathrm{g} / \mathrm{kg}$ and higher.

The first-in-human study was consistent with the lack of Lambda-associated myelosuppression observed in vitro and in cynomolgus monkeys, but the data also highlighted the limitations of the cynomolgus model for estimating human responses. Lambda appeared to be pharmacodynamically more potent in humans, with an equivalent NOEL for $\beta 2 \mathrm{M}$ induction significantly lower than the $5.0 \mu \mathrm{g} / \mathrm{kg}$ estimated from the monkey data.

\section{Phase 1b: HCV GT 1-infected patients}

A three-part, multidose Phase $1 \mathrm{~b}$ proof-of-concept study was undertaken in patients infected with HCV GT 1 to assess the antiviral efficacy and safety of short-term (4 weeks) Lambda administered at five weight-based dose levels between 0.5 and $3.0 \mu \mathrm{g} / \mathrm{kg}$, with ribavirin (Lambda $0.5,0.75,1.5$, or $2.25 \mu \mathrm{g} / \mathrm{kg}$ weekly) or without ( 1.5 or $3.0 \mu \mathrm{g} / \mathrm{kg}$ every 1 or 2 weeks). This study and its results have been fully described elsewhere. ${ }^{28}$ Briefly, over 4 weeks of treatment, Lambda promoted dose-related declines in HCV RNA levels from 
Anderson H. et al: Peginterferon lambda for hepatitis C
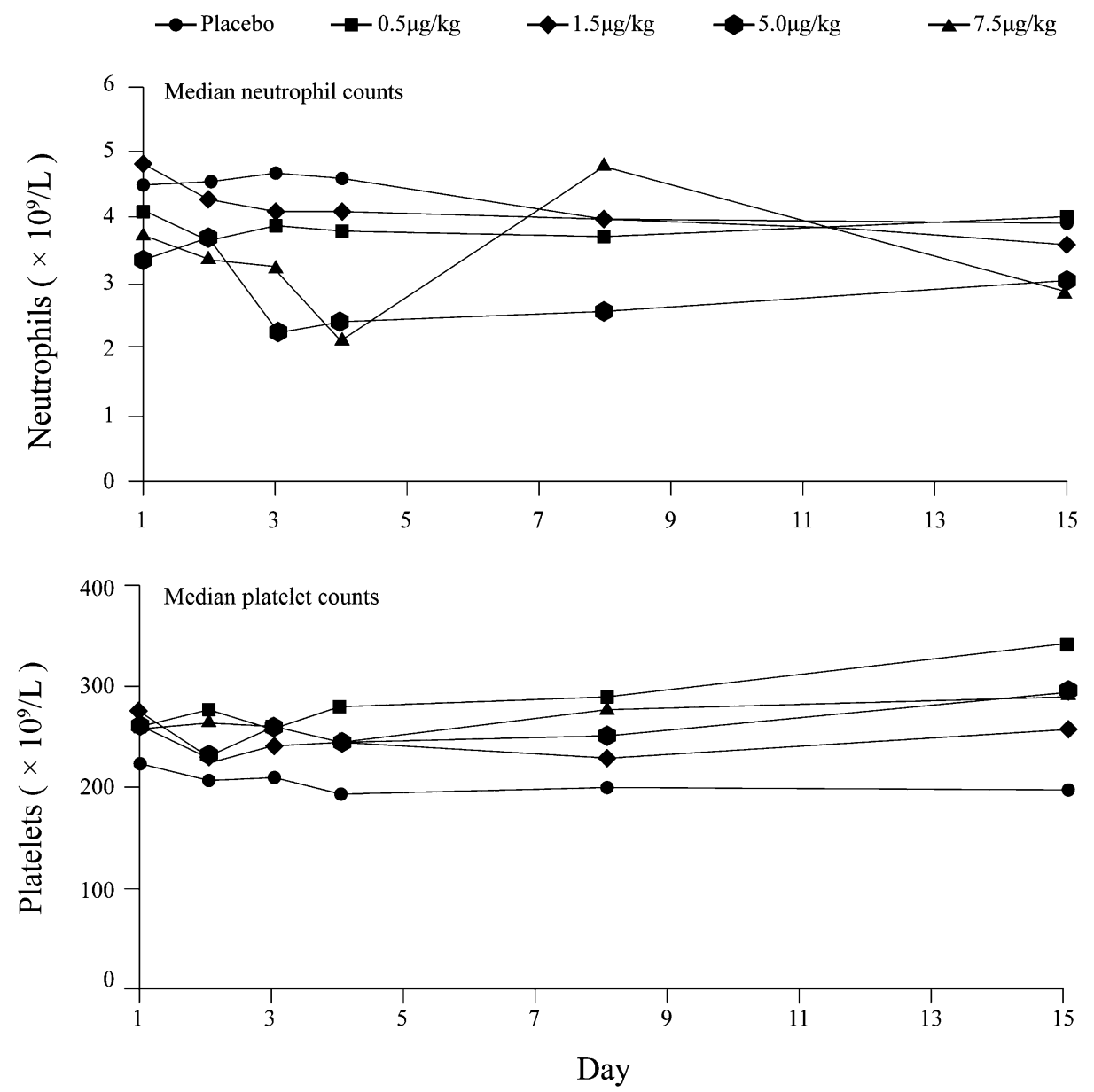

Fig. 4. First-in-human hematologic effects of peginterferon lambda-1a administered as a single dose to healthy volunteers

baseline both as a single agent and with ribavirin, with mean maximal declines ranging from 1.83 to $3.85 \log _{10} \mathrm{IU} / \mathrm{mL}$. No on-treatment declines in neutrophil or platelet counts were observed in patients treated with Lambda plus ribavirin. No change in hemoglobin levels occurred in patients receiving Lambda as a single agent, and mild ribavirin-associated hemoglobin declines under combination treatment did not show any Lambda dose effect. Overall, six patients experienced aminotransferase elevations, with or without bilirubin elevations, of whom four were receiving the highest Lambda dose tested $(3.0 \mu \mathrm{g} / \mathrm{kg})$. Notably, patients with significant alanine aminotransferase elevations displayed high PK exposure to Lambda (area under the concentration curve). Other adverse events were mostly mild or moderate and did not appear to be dose related.

Multidose Lambda PK in these HCV-infected patients were consistent with the single-dose data from healthy volunteers. Dose normalization of the Lambda area under the concentration curve suggested a linear relationship, with no apparent effect of patient weight on exposure observed by linear regression. A continuous relationship was observed between Lambda exposure and decreases in HCV RNA.

In summary, Phase 1 studies demonstrated that treatment with Lambda was associated with rapid, exposure-related antiviral activity, established linear dose-dependent exposure that allowed for simpler fixed dosing to be carried forward into Phase 2 trials, and suggested that Lambda may produce less neutropenia and anemia than historically seen with the type I alfa IFNs. An increased risk of aminotransferase elevations at high Lambda exposures, with or without associated increases in bilirubin levels, was an unexpected outcome in these early human studies that highlighted pharmacodynamic differences between the activity of Lambda in humans and monkeys.

\section{Phase 2a: head-to-head activity against PEG-IFN alfa- $2 a$}

Early Phase 2a development (see Muir et al. ${ }^{35}$ for abstract data) evaluated the PK and head-to-head antiviral efficacy and safety of fixed doses of Lambda plus ribavirin versus an active PEG-IFN alfa-2a plus ribavirin control in treatmentnaive patients with HCV GT $1-4$. Patients $(\mathrm{N}=55)$ received weekly subcutaneous Lambda at $80,120,180$, or $240 \mu \mathrm{g}$ fixed doses in combination with daily ribavirin, or $180 \mu \mathrm{g}$ PEGIFN alfa-2a. On-treatment rates of undetectable HCV RNA at weeks 4 and 12 for the Lambda 120-240 $\mu \mathrm{g}$ arms (week 12, $43-71 \%$ in GT 1 or GT 4 and $80-100 \%$ in GT 2 or GT 3) were at least similar to those of the PEG-IFN alfa-2a control (week $12,40 \%$ and $100 \%$, respectively), whereas rates for Lambda 

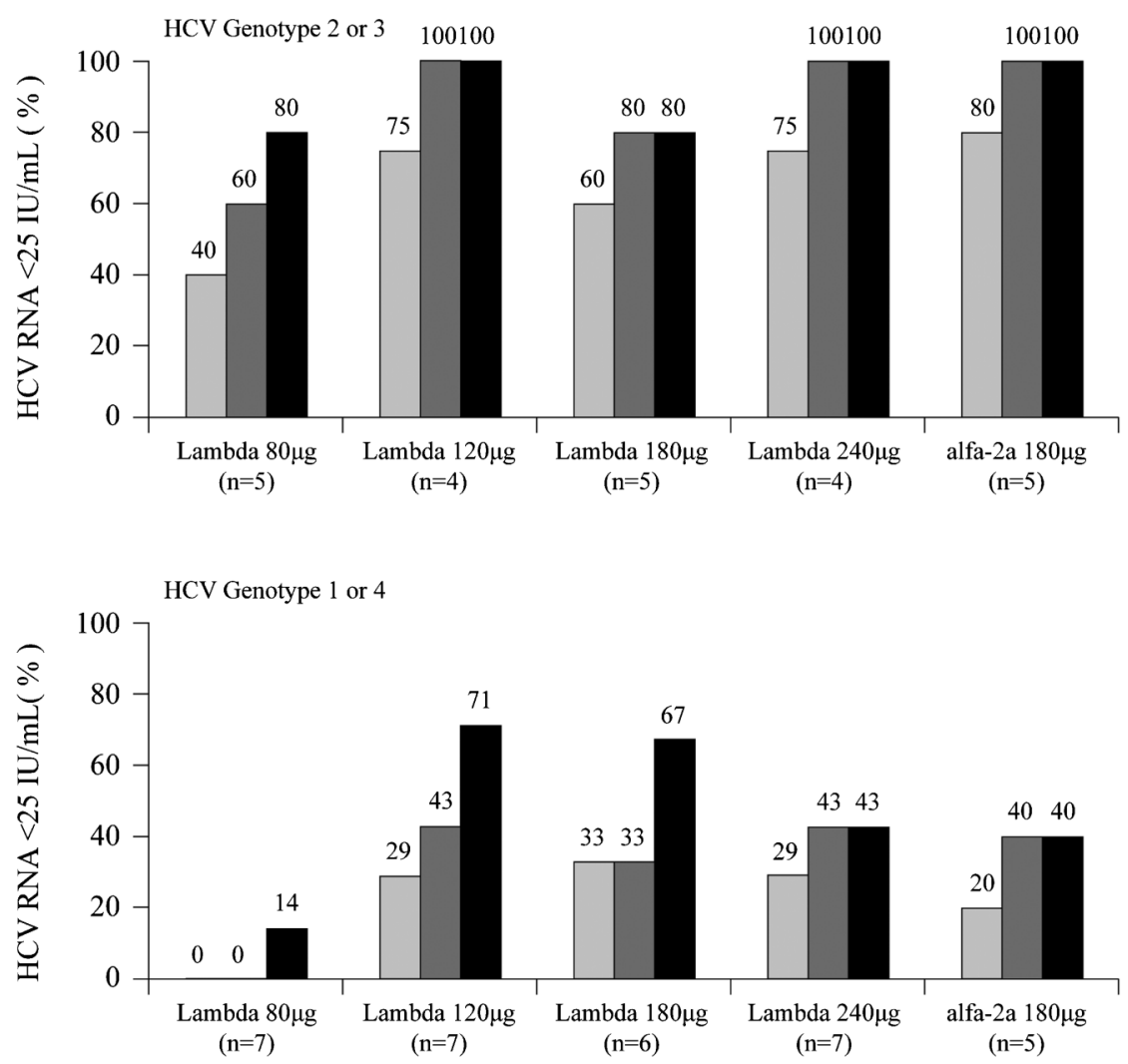

Fig. 5. First head-to-head efficacy data for peginterferon lambda-1a vs peginterferon alfa-2a in a Phase $2 a$ study

$80 \mu \mathrm{g}$ were lower than those of the control, particularly for GT 1 or GT 4 (week 12, 14\%; Fig. 5). Four patients in the higher Lambda dosing arms (three at $240 \mu \mathrm{g}$ and one at $180 \mu \mathrm{g}$ ) met protocol-defined criteria for dose adjustment due to elevated aminotransferase levels. Lambda PK were similar to those observed for weight-based dosing in the Phase $1 \mathrm{~b}$ study. Across 12 weeks, no reduction in mean neutrophil or platelet counts were seen in the Lambda dosing arms, compared with clear reductions in the PEG-IFN alfa-2a arm. On-treatment myalgia across 12 weeks was numerically lower among patients receiving Lambda (six of 45 patients [13\%]) than among those receiving PEG-IFN alfa-2a (four of 10 patients [40\%]).

These early on-treatment Phase $2 a$ data were notable primarily for establishing that the antiviral activity of Lambda against HCV in vivo was not intrinsically inferior to that of the type I therapeutics. The data also established the utility of fixed dosing and the effective dosing range (120-240 $\mu \mathrm{g}$ ) that was carried forward into larger Phase $2 b$ studies, as well as corroborating the minimal impact of Lambda on hematologic parameters that was observed with single-dose and short-term administration in Phase 1 trials.

\section{Conclusions}

We have chosen to restrict the clinical portion of this short review to the early, small-scale Phase 1 and 2a studies that showed both the strengths and limitations of the preclinical models used, and that finally established the case for comparable in vivo anti-HCV activity at fixed doses between Lambda and the established PEG-IFNs. On the basis of the Phase 2a data, a large dose-ranging Phase $2 b$ study was completed (see Zeuzem et al. ${ }^{36}$ and Muir et al. ${ }^{37}$ for abstract data). This study and the numerous subanalyses derived from it, including the derivation of the final $180 \mu \mathrm{g} /$ week Phase 3 dose, are beyond the scope of this manuscript and will be reported elsewhere.

The type III IFNs provide an object lesson in how exploring a biologic niche can sometimes yield new therapeutic insights. In nature, endogenous type III IFNs appear to function primarily as antiviral support for type I IFNs in epithelial cells. Because epithelial membranes in the lung and gut are the primary sites of ingress for many viruses, a second system inducing a type I-like response through an unrelated receptor would likely improve innate immunity to a number of infections. ${ }^{38}$ The role of endogenous type III IFNs in epithelial-like hepatocytes appears to be more complex and less easy to conceptualize. Mouse data have suggested that although the type III system contributes to protection against influenza virus infection in the lung, it does not appear to play a significant role in the liver in strengthening type I-mediated immunity to hepatotropic viruses. ${ }^{39}$ However, it was subsequently discovered that polymorphisms in human $I L 28 B$, in particular the rs12979860 single-nucleo- 
tide polymorphism located $3 \mathrm{~kb}$ upstream of the $I L 28 B$ gene locus, are strongly associated with both spontaneous HCV clearance and the response of HCV GT 1 to exogenous IFN alfa. ${ }^{40-44}$ A CC homozygous GT at the rs12979860 locus is associated with a substantially (approximately two- to threefold) elevated probability of a sustained GT 1 virologic response to IFN alfa plus ribavirin compared with a $\mathrm{CT}$ or homozygous $\mathrm{TT}$ GT, and allelic carriage at this site shows marked racial differences, which may help to explain known differences in HCV treatment outcomes among populations. ${ }^{40,41}$ Although the mechanism of this effect is not yet understood, its existence suggests a role for the type III axis in human hepatitis infection that goes beyond a redundant recapitulation of type I activity.

Whatever its precise endogenous role in viral hepatitis, the potential for IFN- $\lambda 1$ as a modified exogenous therapeutic is now established. As a drug, the pegylated molecule has the same once-weekly dosing as the pegylated type I therapeutics, but benefits from a remarkable reduction in several of their signature toxicities as a result of its more focused activity in the target organ. ${ }^{36,37,45}$ The aminotransferase elevations sometimes observed at high Lambda exposuresmost often at doses above the fixed $180 \mu \mathrm{g} /$ week carried into ongoing studies-have been manageable in Phase $2 b$ trials by dose adjustments or discontinuation. Given its safety and efficacy profile through Phase 2 development, Lambda may find future utility in combination regimens with direct-acting antiviral agents. Phase 3 evaluation of Lambda with and without such agents is currently underway in HCV GT 1-4, and further clinical data are awaited with interest.

\section{Conflict of interest}

All authors are, or were at the time of the work described, employees of ZymoGenetics/Bristol-Myers Squibb and may be stockholders thereof.

\section{Writing assistance}

Editorial assistance with the drafting of this manuscript was provided by Nick Fitch, PhD, of ArticulateScience (London, UK) with funding from Bristol-Myers Squibb.

\section{Author contributions}

Design, performance, analysis, and/or interpretation of the ZymoGenetics-based studies (HA, JM, JF, SED, KK, DMM, DH, $\mathrm{JLH})$, initial drafting of the manuscript ( $\mathrm{HA}, \mathrm{JLH})$, text revision and approval (HA, JM, JF, SED, KK, DMM, DH, JLH).

\section{Acknowledgments}

The authors would like to acknowledge the essential contributions of their many colleagues and former colleagues at ZymoGenetics and Bristol-Myers Squibb-particularly Paul Sheppard, Mark Snavely, Kelly Byrnes-Blake, Rafael Ponce, Robert West, Hong Liu, Tom Bukowski, Megan Lantry, Susan Pederson, and David Fontana-without whose work Lambda could never have been developed.

\section{References}

[1] Lavanchy D. The global burden of hepatitis C. Liver Int 2009;29:74-81.
[2] World Health Organization. Prevention \& control of viral hepatitis infection: framework for global action. Available at: www.who.int/csr/disease/hepatitis/ GHP_framework.pdf, accessed October 2013.

[3] Sulkowski MS, Cooper C, Hunyady B, Jia J, Ogurtsov P, Peck-Radosavljevic M, et al. Management of adverse effects of Peg-IFN and ribavirin therapy for hepatitis C. Nat Rev Gastroenterol Hepatol 2011;8:212-223.

[4] Ghany M, Nelson DR, Strader DB, Thomas DL, Seeff LB. An update on treatment of genotype 1 chronic hepatitis $C$ virus infection: 2011 practice guidelines by the American Association for the Study of Liver Diseases. Hepatology 2011;54:1433-1444.

[5] Qin H, Li H, Zhou X, Feng F, Shen Y, Tan H, et al. Safety of telaprevir for chronic hepatitis $\mathrm{C}$ virus infection: a meta-analysis of randomized controlled trials. Clin Drug Investig 2012;32:665-672.

[6] Maasoumy B, Port K, Markova AA, Serrano BC, Rogalska-Taranta M, Sollik L, et al. Eligibility and safety of triple therapy for hepatitis C: lessons learned from the first experience in a real world setting. PLoS One 2013;8:e55285.

[7] Teixeira R, Nascimento Yde A, Crespo D. Safety aspects of protease inhibitors for chronic hepatitis C: adverse events and drug-to-drug interactions. Braz J Infect Dis 2013;17:194-204

[8] Sheppard P, Kindsvogel W, Xu W, Henderson K, Schlutsmeyer S, Whitmore TE, et al. IL-28, IL-29 and their class II cytokine receptor IL-28R. Nat Immunol 2003;4:63-68.

[9] Kotenko SV, Gallagher G, Baurin VV, Lewis-Antes A, Shen M, Shah NK, et al. IFN- $\lambda s$ mediate antiviral protection through a distinct class II cytokine receptor complex. Nat Immunol 2003;4:69-77.

[10] Donnelly RP, Kotenko SV. Interferon-lambda: a new addition to an old family. J Interferon Cytokine Res 2010;30:555-564.

[11] Kotenko SV. IFN- $\lambda$ s. Curr Opin Immunol 2011;23:583-590.

[12] Donnelly RP, Dickensheets $\mathrm{H}, \mathrm{O}^{\prime}$ Brien TR. Interferon-lambda and therapy for chronic hepatitis $C$ virus infection. Trends Immunol 2011;32:443-450.

[13] Doyle SE, Schreckhise H, Khuu-Duong K, Henderson K, Rosler R, Storey H, et al. Interleukin-29 uses a type 1 interferon-like program to promote antiviral responses in human hepatocytes. Hepatology 2006;44:896-906.

[14] Witte K, Gruetz G, Volk HD, Looman AC, Asadullah K, Sterry W, et al. Despite IFN- $\lambda$ receptor expression, blood immune cells, but not keratinocytes or melanocytes, have an impaired response to type III interferons: implications for therapeutic applications of these cytokines. Genes Immun 2009;10:702714.

[15] Sommereyns C, Paul S, Staeheli P, Michiels T. IFN-lambda (IFN- $\lambda$ ) is expressed in a tissue-dependent fashion and primarily acts on epithelial cells in vivo. PLoS Pathog 2008;4:e1000017.

[16] Marcello T, Grakoui A, Barba-Spaeth G, Machlin ES, Kotenko SV, MacDonald $M R$, et al. Interferons $\alpha$ and $\lambda$ inhibit hepatitis $C$ virus replication with distinct signal transduction and gene regulation kinetics. Gastroenterology 2006; 131:1887-898.

[17] Yin Z, Dai J, Deng J, Sheikh F, Natalia M, Shih T, et al. Type III IFNs are produced by and stimulate human plasmacytoid dendritic cells. J Immuno 2012;189:2735-2745.

[18] Byrnes-Blake KA, Pederson S, Klucher KM, Anderson-Haley M, Miller DM, Lopez-Talavera JC, et al. Pharmacokinetics and pharmacodynamics of pegylated interferon lambda-1 in cynomolgus monkeys. J Interferon Cytokine Res 2012;32:198-206.

[19] Robek MD, Boyd BS, Chisari FV. Lambda interferon inhibits hepatitis B and C virus replication. J Virol 2005;79:3851-3854.

[20] Zhu H, Butera M, Nelson DR, Liu C. Novel type I interferon IL-28A suppresses hepatitis C viral RNA replication. Virol J 2005;2:80.

[21] Baker DE. Pegylated interferons. Rev Gastroenterol Disord 2001;1:87-99.

[22] Kozlowski A, Charles SA, Harris JM. Development of pegylated interferons for the treatment of chronic hepatitis C. BioDrugs 2001;15:419-429.

[23] Grace MJ, Cutler D. Pegylating IFNs at his-34 improves the in vitro antiviral activity through the JAK/STAT pathway. Antivir Chem Chemother 2004;15: 287-297.

[24] Grace M, Youngster S, Gitlin G, Sydor W, Xie L, Westreich L, et al. Structural and biologic characterization of pegylated recombinant IFN-alpha2b. J Interferon Cytokine Res 2001;21:1103-1115.

[25] Foser S, Schacher A, Weyer KA, Brugger D, Dietel E, Marti S, et al. Isolation, structural characterization, and antiviral activity of positional isomers of monopegylated interferon alpha-2a (PEGASYS). Protein Expr Purif 2003;30: 78-87.

[26] Harris JM, Herati RM. Preparation and use of poly-ethylene glycol propionaldehyde. US Patent 5,252,714. 1993.

[27] Byrnes-Blake KA, Freeman JA, Rapalus L, Pederson S, Fontana D, LopezTalavera JC, et al. Pharmacokinetics of peg-interferon lambda (pegIFN $\lambda$ ) following fixed dosing in treatment-naive hepatitis $\mathrm{C}$ subjects (single-dose interim data from a dose-ranging Phase 2a study). Hepatology 2010;52: 720A-721A.

[28] Muir AJ, Shiffman ML, Zaman A, Yoffe B, de la Torre A, Flamm S, et al. Phase 1 b study of pegylated interferon lambda 1 with or without ribavirin in patients with chronic genotype 1 hepatitis C virus infection. Hepatology 2010;52: 822-832. 
[29] Hausman DF, Freeman JA, Souza SM, Nesterov IA, Zhang T. A Phase 1, randomized, blinded, placebo-controlled, single-dose, dose-escalation study of PEG-interferon lambda (PEG-rIL-29) in healthy subjects. Global Antiviral J 2007;3(Suppl 2):71-72.

[30] Maher SG, Sheikh F, Scarzello AJ, Romero-Weaver AL, Baker DP, Donnelly RP, et al. IFN- $\alpha$ and IFN- $\lambda$ differ in their antiproliferative effects and duration of JAK/STAT signaling activity. Cancer Biol Ther 2008;7:1109-1115.

[31] Freeman JA, Zhang T, Holdren MS, Hausman DF. PEG-interferon lambda (PEG-rIL-29): translation of in vitro preclinical data to clinical results. J Hepatol 2008;48(Suppl 2):S294.

[32] Bannai H, Tatsumi M, Kohase M, Onishi E, Yamazaki S. Pharmacokinetic study of a human recombinant interferon (Re-IFN-alpha A) in cynomolgus monkeys by $2^{\prime}-5^{\prime}$ oligoadenylate synthetase assay. Jpn J Med Sci Biol 1985; 38: $113-124$

[33] Bannai $\mathrm{H}$. Comparison of pharmacokinetic behaviors of two human interferons (Lb-IFN-alpha and Re-IFN-alpha A) in cynomolgus monkeys by $2^{\prime}-5^{\prime}$ oligoadenylate synthetase assay. Jpn J Med Sci Biol 1986;39:185-198.

[34] Miller DM, Klucher KM, Freeman JA, Hausman DF, Fontana D, Williams DE. Interferon lambda as a potential new therapeutic for hepatitis C. Ann N Y Acad Sci 2009;1182:80-87.

[35] Muir AJ, Lawitz E, Ghalib RH, Sussman NL, Anderson F, Everson GT, et al. Pegylated interferon lambda (PEG-IFN $\lambda$ ) Phase 2 dose-ranging, activecontrolled study in combination with ribavirin (RBV) for treatment-naïve HCV patients (genotypes 1, 2, 3 or 4): safety, viral response, and impact of IL-28B host genotype through week 12. Hepatology 2010;52:715A.

[36] Zeuzem S, Arora S, Bacon B, Box T, Charlton M, Diago M, et al. Peginterferon lambda-1a compared to peginterferon alfa-2a in treatment-naive patients with HCV genotype 2 or 3: first SVR24 results from EMERGE Phase IIB. J Hepatol 2012;56(Suppl 2):S5-S6.

[37] Muir A, Hillson J, Gray T, Xu D, Ishak L, Freeman J, et al. Peginterferon lambda-1a (Lambda) compared to peginterferon alfa-2a (alfa) in treatment- naive patients with HCV genotypes 1 or 4: SVR24 results from EMERGE Phase 2b. Hepatology 2012;56(Suppl):299A.

[38] Mordstein M, Neugebauer E, Ditt V, Jessen B, Rieger T, Falcone V, et al. Lambda interferon renders epithelial cells of the respiratory and gastrointestinal tracts resistant to viral infections. J Virol 2010;84:5670-5677.

[39] Mordstein M, Kochs G, Dumoutier L, Renauld JC, Paludan SR, Klucher K, et al. Interferon- $\lambda$ contributes to innate immunity of mice against influenza $A$ virus but not against hepatotropic viruses. PLoS Pathog 2008;4:e1000151.

[40] Ge D, Fellay J, Thompson AJ, Simon JS, Shianna KV, Urban TJ, et al. Genetic variation in IL28B predicts hepatitis C treatment-induced viral clearance. Nature 2009;461:399-401.

[41] Thomas DL, Thio CL, Martin MP, Qi Y, Ge D, O'Huigin C, et al. Genetic variation in IL28B and spontaneous clearance of hepatitis C virus. Nature 2009;461: 798-801.

[42] Tanaka Y, Nishida N, Sugiyama M, Kurosaki M, Matsuura K, Sakamoto N, et al. Genome-wide association of IL28B with response to pegylated interferon-alpha and ribavirin therapy for chronic hepatitis C. Nat Genet 2009;41:1105-1109.

[43] Suppiah V, Moldovan M, Ahlenstiel G, Berg T, Weltman M, Abate ML, et al. IL28B is associated with response to chronic hepatitis $C$ interferon- $\alpha$ and ribavirin therapy. Nat Genet 2009;41:1100-1104.

[44] Thompson AJ, Muir AJ, Sulkowski MS, Ge D, Fellay J, Shianna KV, et al. Interleukin-28B polymorphism improves viral kinetics and is the strongest pretreatment predictor of sustained virologic response in genotype 1 hepatitis C virus. Gastroenterology 2010;139:120-129.

[45] Muir AJ, Srinivasan S, Sapra S, Dimitrova D, Hillson JL, Lopez-Talavera JC. Peginterferon lambda-1a is less likely to induce clinically significant neuropsychiatric symptoms during the treatment of chronic hepatitis $\mathrm{C}$ virus infection, compared to peginterferon alfa-2a. Hepatology 2012;56(Suppl): 578A-579A. 